\title{
Osteopetrosis in siblings
}

\author{
Ziba Mosayebi ${ }^{1} \cdot$ Hadi Mirfazaelian ${ }^{2} \cdot$ Bijan Khademi $^{3} \cdot$ Neda Bagheri $^{4}$. \\ Yahya Daneshbod ${ }^{4,5}$
}

Received: 21 September 2015/Accepted: 5 October 2015/Published online: 3 November 2015 (C) SIMI 2015

A 14-year-old girl presented with gradual visual loss. It started gradually during early childhood, eventuating in complete blindness. The past medical history was positive for multiple fractures. On physical examination, there was bulging on the left side of the mandible. Orthopantogram revealed increased density and sclerosis of the skull base with a "snow white" appearance (Fig. 1a). Neural foramina were encroached, and the left mandibular body showed periosteal reaction and sequestra formation. Multiple axial

Yahya Daneshbod

daneshbk@yahoo.com; y@daneshbod.com

1 Department of Pediatrics, Tehran University of Medical Sciences, Tehran, Iran

2 Department of Emergency Medicine, Tehran University of Medical Sciences, Tehran, Iran

3 Department of Head and Neck Surgery, Shiraz School of Medicine, Shiraz, Iran

4 Department of Surgical Pathology, Dr Daneshbod Pathology Laboratory, Shiraz, Iran

5 Department of Cytopathology, Dr Daneshbod Pathology Laboratory, Shiraz, Iran and coronal computed tomography images after administration of IV contrast were taken which show increased bone density in the base of skull representing osteopetrosis. In the left mandibular ramus, an inhomogenous density associated with periosteal elevation and subperiosteal bone formation with soft tissue swelling surrounding it is noted with extension to left side of mandibular body (Fig. 1b, c). One of her brothers, a 15-year-old boy had facial swelling and pain since he was 9 . He had a history of multiple dental extractions due to severe dental caries. Over the past 3 years, he received multiple courses of antibiotics for a diagnosis of maxillary sinusitis. He also complained of hearing and visual problems. Coronal computed tomography from nasal cavity and facial bone showed destruction of both the nasal antrum accompanied with sequestration formation and sclerosis of the maxillary, zygomatic, and pterygoid plates (Fig. 1d). Osteopetrosis, is caused from dysfunction of osteoclasts [1]. There are three main clinical variants in humans: infantile, intermediate, and adult [2]. The infantile variant is often associated with severe obliteration of the bone marrow that results in anemia and thrombocytopenia, neuropathies related to cranial nerve entrapment, and failure to thrive and growth retardation. In the maxilla and mandible, osteopetrosis is often complicated by osteomyelitis, which is refractory in most cases [2, 3]. The intermediate type exhibits abnormal dentition, jaw osteomyelitis, cranial nerve deficits, and sometimes local bone swelling. These manifestations tend to occur at the end of the first decade of life. The adult variant is symptom free, and usually the diagnosis is established by imaging or family history. The cases reported here were regarded as consistent with an intermediate form of osteopetrosis judging from the onset and clinical progress of the disease. Segmental resection of the mandible including sequestrectomy was performed in the second patient. 

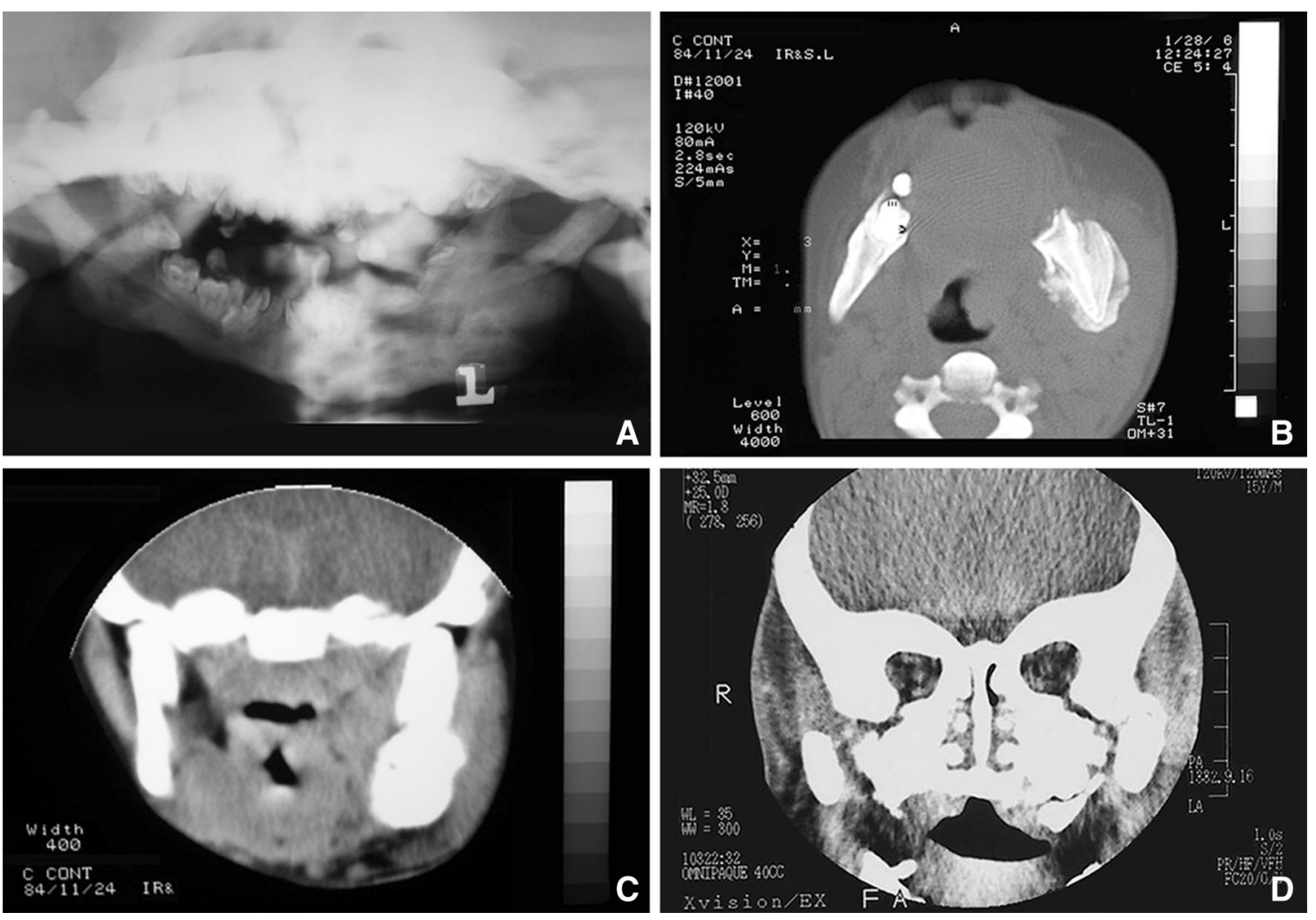

Fig. 1 a Orthopantogram showed "snow white" appearance of skull base, encroached neural foramina, periosteal reaction, and sequestra formation in left mandibular body. b, c CT imaging with IV contrast reveals increased bone density in the base of skull and inhomogenous

density associated with subperiosteal bone formation with periosteal elevation and soft tissue swelling in mandibular body. d Coronal CT imaging showed destruction of both nasal antra and sequestration formation and sclerosis of maxilla, zygoma, and pterygoid plates

\section{Compliance with ethical standards}

\section{References}

Conflict of interest The authors declare that they have no conflict of interest

Statement of human and animal rights All procedures performed in studies involving human participants were in accordance with the ethical standards of the institutional and/or national research committee and with the 1964 Helsinki declaration and its later amendments or comparable ethical standards. This article does not contain any studies with animals performed by any of the authors.

Informed consent Informed consent was obtained from patients or/ and guardians in this study.

1. Albers-Schönberg HE (1904) Röntgenbilder einer seltenen Knockenerkrankung. Munch Med Wochenschr 51:365-368

2. Shapiro F (1993) Osteopetrosis: current clinical considerations. Clin Orthop Relat Res 294:34-44

3. Loria-Cortes R, Quesada-Calvo E, Cordero-Chaverri C (1977) Osteopetrosis in children: a report of 26 cases. J Pediatr 91:43-47 